\title{
Culture and Mobility Determine the Importance of Similarity in Friendship
}

\author{
Angela J. Bahns \\ Wellesley College, USA \\ Juwon Lee \\ Carnegie Mellon University, USA \\ Christian S. Crandall \\ University of Kansas, USA
}

\section{This is the author's accepted version of the article:}

Bahns, A. J., Lee, J., \& Crandall, C. S. (2019). Culture and mobility determine the importance of similarity in friendship. Journal of Cross-Cultural Psychology. Available online at https://doi.org/10.1177/0022022119852424

\section{Author Note}

Angela J. Bahns, Department of Psychology, Wellesley College, USA; Juwon Lee, Department of Psychology, Carnegie Mellon University, USA; Christian S. Crandall, Department of Psychology, University of Kansas, USA.

We thank Laura Allen, Amanda Campbell, Myeongyun Choi, Ashley Currie, Simonetta Gramolini, Victoria Grandsoult, Alex Jacobson, Juwon Lee, Aaron Neis, Annie Park, Vance Rexwinkle, Monica Setaruddin, Kyle Speakman, and Heather Tice for their help in collecting the field data.

Correspondence concerning this article should be addressed to Angela Bahns, abahns@wellesley.edu. Research materials are available at https://osf.io/pm8c4/.

\section{Funding}

This research was supported by the Wellesley College Office of the Provost and Dean of the College. 


\begin{abstract}
We test whether the ability to start friendships — and end them—determines what we look for in friends. We hypothesized that individual differences in beliefs about relationship choice would translate into differences in similarity of relationship partners. To test the constructs across their spectrum, we sampled both American and Korean relationships $(N=1,603)$. Study 1 fieldsampled naturally-occurring relationship pairs in the USA and Korea; independent self-construal was positively correlated with relational mobility and similarity within relationship pairs. Using the entire data set, relational mobility was positively correlated with similarity, but the two were negatively correlated within nations. Study 2 identified two components of relational mobility, Entry and Exit. Easy entry to relationships correlated with high importance of similarity; easy exit from relationships correlated with low importance of similarity. An experiment (Study 3) manipulating both ease of entry to and exit from friendship found the belief that people are relatively free to end an unsatisfying friendship reduced the importance of similarity for friendship selection.
\end{abstract}

Keywords: friendship, similarity-attraction, independent self-construal, relational mobility, choice-based selection 


\section{Culture and Mobility Determine the Importance of Similarity in Friendship}

People like those who are like them. This simple but robust finding, known as the similarity-attraction effect, plays a central role in relationships research (e.g., Byrne, 1971; Jones, Pelham, Carvallo, \& Mirenberg, 2004; Newcomb, 1956). Research has shown that friendships and other close relationships are often characterized by similarity of partners' attitudes, values, behaviors, ethnicity, age, and gender (Graziano \& Bruce, 2008; McPherson, Smith-Lovin, \& Cook, 2001; Titzmann, Silbereisen, \& Mesch, 2012). Cross-cultural research has found the magnitude of the similarity-attraction effect tends to be larger in Western than East Asian samples (Heine, Foster, \& Spina, 2009; Heine \& Renshaw, 2002; Schug, Yuki, Horikawa, \& Takemura, 2009). We propose these findings may be linked to cultural differences in beliefs about relationship choice.

The goal of the current research was to explore how differences in beliefs about choice and a related sense of relational mobility affect the degree of similarity within relationships. Relational mobility reflects the amount of opportunity people have to form new relationships and terminate existing ones (Thomson et al., 2018). In this paper we examined the relationships between choice, mobility, and similarity-based selection strategies in the United States of America (USA) and South Korea. We sampled from these two countries to ensure variability on the constructs. Geographical lines alone are not what determine cultural comparisons (Betancourt, Hardin, \& Manzi, 1992); culture can also be studied by drawing comparisons across internal representations of choice, relationships, values, and construals.

The cultural values of individualism and collectivism refer to the extent to which people are assumed to be independent of, or bound to, one another (Triandis, 1995). Individualism portrays people as fundamentally separate, or independent, from others, rendering them free to 
make their choices based on their personal preferences. Collectivism portrays people as inherently connected and obligated to others. Because the USA is substantially more individualistic than Korea (Kim, Park, \& Suzuki, 1990; Oyserman et al., 2002), we predict there should be a greater sense of relationship choice in the USA than Korea. Indeed previous research suggests that relational mobility is higher in North American societies than in East Asian societies (Triandis, 1995; Wiseman, 1986).

Cultural differences may also manifest as macro level variations in individuals' values, beliefs and construals. Independent self-construal reflects the extent to which the self is viewed as an independent agent (Markus \& Kitayama, 1991); it reflects an understanding of the self as autonomous and agentic. In the USA, cultural messages foster "an understanding of the self as autonomous, freedom seeking, defined by one's unique assemblage of interests, pursuits, beliefs, and attitudes, and obligated to no one and nothing except by one's own choice" (Cross \& Gore, 2004, p. 230). We propose that independent self-construal encourages the belief that close relationships are formed to satisfy personal preferences. Because similarity in friendships is preferred and sought after, independent self-construal should predict both relational mobility and similarity within friendships.

Similarity in close relationships has two major competing accounts. A social influence account predicts that friends and partners become more similar to one another as their relationship develops (Bourgeois \& Bowen, 2001; Cullum \& Harton, 2007). By contrast, a selection account assumes that partners choose one another based on pre-existing similarities (Urberg, Degirmencioglu, \& Tolson, 1998; Watson, Klohnen, Casillas, Simms, \& Haig, 2004). Consistent with prior work, we propose that as perceived choice in relationships increases, 
similarity will also increase, as posited by the selection account of it (Bahns, Pickett, \& Crandall, 2012).

In a field study of American friendship pairs, Bahns, Crandall, Gillath, and Preacher (2017) found strong evidence for social selection and surprisingly little evidence for social influence in accounting for similarity within friends. Friends were similar upon meeting and similarity did not increase with time, closeness, or discussion. In the current research we argue that selection is most likely to lead to similarity in cultural contexts that offer relationship choice. In environments with little choice, lower levels of similarity emerge (Bahns et al., 2012; Ishiguro, 2011; Schug et al., 2009). The two markers of relationship choice we explore in this paper are independent self-construal and relational mobility.

The link between independent self-construal and relational mobility is made apparent in decisions about relationship initiation and dissolution. People in individualistic cultures acknowledge relationships are necessary for individuals to thrive, but also recognize the maintenance of social relations may be costly (Kagitcibasi, 2005; Oyserman, 1993). We propose that people high in independent self-construal may feel more entitled to terminate existing relationships and initiate new relationships in accordance with their personal preferences and goals.

Relational mobility tends to be higher in North American societies than East Asian societies (Schug et al., 2009; cf. Zhang \& Li, 2014). With high relational mobility, people believe they are free to form new relationships or leave existing relationships as they see fit. With low relational mobility, the initiation and dissolution of relationships is more dependent on external constraints. Prior research links relational mobility to similarity of friendship choices. For example, Schug et al. (2009) found that American students perceived themselves as more 
similar to their friends than Japanese students; this difference was mediated by relational mobility.

\section{Overview}

In three studies we compared American and Korean relationship partners on similarity, relational mobility, and relationship choice. We expected that individual differences in beliefs about agency and relationship choice would translate into differences in similarity within close relationships.

In Study 1, we field-sampled pairs in public settings in the USA and Korea and examined the relationships between independent self-construal, relational mobility, and similarity of relationship partners' attitudes and behaviors. In Study 2, we used self-report methods and examined the relationships between belief in friendship choice, the separate entry and exit components of relational mobility, and the rated importance and perceived similarity of friends. In Study 3, we manipulated the relative ease of relationship entry and exit and measured the effects of each on the importance of similarity in a friend.

\section{Study 1}

Study 1 used a free-range dyad harvest field method (Crandall, Schiffhauer, \& Harvey, 1997), to sample naturally-occurring relationship pairs in the USA and Korea. We measured individual differences in self-construal to reflect beliefs about agency and choice in relationships. We predicted that Americans would be higher than Koreans in independent self-construal (Markus \& Kitayama, 1991), that relational mobility would be higher in the USA than in Korea, and that greater relational mobility would in turn predict similarity-based selection. Thus, we predicted that relationship pairs would be more similar in the USA as compared to Korea. In 
addition, we hypothesized a mediational model in which the effect of independent self-construal ${ }^{1}$ on within-pair similarity would be mediated by relational mobility.

\section{Method}

\section{Participants}

Research assistants approached naturally-occurring pairs in public spaces in four different cities in the United States (Lawrence, KS, $N=80$ pairs; Iowa City, IA, $N=28$ pairs; Chicago, IL, $N=49$ pairs; and New York, NY, $N=96$ pairs) and two different cities in South Korea (Seoul, $N=75$ pairs; Gongju, $N=70$ pairs). All samples included both same-sex and opposite-sex pairs. Population statistics for each of the six cities are reported in Table 1.

American sample. The American sample included 253 pairs, or 506 unique participants (67 female pairs, 43 male pairs, 123 female-male pairs, and 20 pairs that did not report sex). Pair members ranged from $14-78$ years old $(M=30.79, M d=26, S D=13.53)$. Pair members' reports of relationship length ranged from very short ( $\mathrm{MIN}=7.5$ days) to long (MAX=54 years; $M=93.86$ months, $M d=48$ months, $S D=119.70$, skew=2.22). The majority $(53.5 \%)$ of the sample identified their relationship as a friendship (friends, 13\%; very good friends, 19.6\%; best friends, 20.9\%); $3.6 \%$ identified as acquaintances, $24.7 \%$ as dating partners, $15.4 \%$ as married, $1.6 \%$ as family, and $1 \%$ reported that they barely knew one another.

Korean sample. The Korean sample included 145 pairs, or 290 unique participants (59 female pairs, 21 male pairs, 59 female-male pairs, and 6 pairs that did not report sex). Pair members ranged from 16-49 years old $(M=23.83$, median=22, $S D=5.97)$. Pair members' reports of relationship length ranged from very short ( $\mathrm{MIN}=1$ hour) to long (MAX=36 years; $M=52.85$ months, median=36 months, $S D=62.16$, skew=2.73). The majority $(58.3 \%)$ of the sample

\footnotetext{
${ }^{1}$ Relational self-construal was also measured. It was unrelated to relational mobility and within-pair similarity and thus excluded from the analysis. Details are available in the online supplements.
} 
identified their relationship as a friendship (friends, 7.6\%; very good friends, $16.6 \%$; best friends, $34.1 \%) ; 9.7 \%$ identified as acquaintances, $26.2 \%$ as dating partners, $2.1 \%$ as married, $0.7 \%$ as family, and $2.8 \%$ reported that they barely knew one another.

\section{Recruitment Procedure}

Pair recruitment in the USA was done by American undergraduate research assistants trained by the authors and by Korean researchers in Korea who received online training from the authors. Pairs were defined as groups of exactly two people who were already interacting with one another before the researcher approached. Researchers went to various locations where people were commonly found in public, as appropriate in each country. When more than one pair was present in a location, researchers approached pairs randomly (aided by a sheet of compass points labeled in random order). Potential participants were told the study was about "how people who know each other are similar or different," and told their answers would remain anonymous. Pairs were explicitly asked to not look at one another's questionnaire and to not discuss their answers until after they handed the questionnaires back to the experimenter.

\section{Materials}

The questionnaire included three cultural variables, six relationship variables, twelve attitude items, three behavior items, and participant age and gender. For Korean participants, the questionnaire was translated into Korean and verified using the back translation method.

Cultural variables. Three items adapted from Thomson et al.'s (2018) relational mobility scale measured relational mobility (USA $\alpha=.57$; Korea $\alpha=.58)^{2}$. The items were "In this

\footnotetext{
${ }^{2}$ In order to keep the questionnaire sufficiently short so as to encourage voluntary participation, our field method dictated that we construct very short versions of the relational mobility and independent and relational self-construal scales. In a separate sample $(N=95)$ that filled out the relational mobility scale (provided by Joanna Schug), our shortened scale correlates $r=.76$ with the entire scale.
} 
city it is easy to meet new people," "People in this city can choose who they interact with," and "People in this city have few chances to meet new people" [reversed]. Items were chosen to reflect opportunity and choice in forming new relationships. Higher scores reflect greater mobility.

Two items (USA $r=.20, \mathrm{p}<.0005$; Korea $r=.10, p=.08$ ) from Singelis's (1994) independent self-construal scale measured individual differences in independent self-construal. The items were "I enjoy being unique and different from others in many respects" and "I am the same person at home that I am at work or school." Higher scores reflect greater independent self-construal.

Cultural variables were measured on 1 (strongly disagree) to 7 (strongly agree) Likerttype scales.

Similarity of attitudes and behaviors. We measured similarity within-pairs across a variety of social attitudes and health behaviors. We chose items for which we expected to observe sufficient variability to detect differences in within-pair similarity and items that were likely to be considered important for relationship partners to share in both cultural contexts. This judgment was made by consultation with Korean researchers, including one of the authors.

Seven attitude items included political views (adapted for each country), endorsement of the Protestant work ethic, belief that the USA should be dominant in all its relations with other countries, support for the current government's approach to relationships with North Korea, the importance of the institution from which a person receives their college degree, the importance of religion, and whether or not abortion should remain legal $(1=$ Strongly disagree, $7=$ Strongly agree). 
Attitudes toward five social groups (people from South Korea, people from North Korea, people from the USA, fat people, gay men) were assessed with single-item feeling thermometers (1=Very negative, $7=$ Very positive $)$.

Three items assessed frequency of health-related behaviors. The items were, "How often do you smoke tobacco?" "How often do you consume alcohol to get drunk?" and "How often do you exercise each week?" $(1=$ Not at all, $7=$ A lot $)$.

We created a similarity index for each pair - the sum of absolute differences between pair members' attitude and behavior scores for all 15 attitudes and behaviors. Because difference scores are affected by sample variance, we first tested whether the variances of attitudes and behaviors were different in the USA and Korea. Levene's test for equality of variances indicated significant differences between the two countries on 10 of 15 variables. Prior to calculating the similarity index, attitude and behavior variables were rescaled by within-country variances. ${ }^{3}$

Relationship variables. Several relationship variables were measured to compare the nature of relationships sampled in each nation. Relationship type was assessed with the item "My relationship with this person is best described as" (Barely Know, Acquaintances, Friends, Very Good Friends, Best Friends, Dating Partners, Married, Family). Interpersonal liking was measured with the item "How much do you like this person?" (1=Not at all, $7=$ Very much). Length of relationship (“How long have you known the person you are with right now?") was measured in months. Hours spent together was measured with the item "On average, how many hours per week do you spend with this person?" Closeness was measured with the item "How close are you to this person?" (1=Not close at all, 7=Very close). Disclosure was measured with

\footnotetext{
${ }^{3}$ We used the observed sample variances in each country and did not incorporate uncertainty into the variance estimates.
} 
the item "Do you feel that you can confide in this person about virtually everything?" $(1=$ No, strongly disagree, $7=$ Yes, strongly agree).

\section{Results and Discussion}

\section{Tests of Between-Country Differences}

Descriptive statistics for the six samples on all measures are presented in Table 1.

Between-country comparisons are reported in Table 2. As hypothesized, relationship pairs were more similar (as indicated by lower discrepancy scores on the similarity index) in the USA compared to Korea. ${ }^{4}$ In addition, Americans scored substantially higher on independent selfconstrual and perceived greater relational mobility than Koreans. It is interesting to note that for independent self-construal even the highest scoring Koreans were below the mean for the American sample.

There were some differences in the nature of the relationships our field method captured in each nation. On average, participants were older and relationship partners had known each other for longer in the USA compared to Korea. In addition, pairs reported liking each other significantly more, spending more time together, and disclosing more personal information to one another in the USA compared to Korea. ${ }^{5}$ Since associating with someone you like, spending time with someone, and self-disclosure are all products of personal choice (Derlega \& Chaikin, 1977; Schug, Yuki, \& Maddux, 2010), these findings are consistent with our expectation that there would be greater relational mobility in the USA than Korea.

\footnotetext{
${ }^{4}$ As an alternative analysis strategy we calculated intraclass correlation coefficients (ICCs) for indistinguishable dyads (Kenny, Kashy, \& Cook, 2006). Across all twelve attitudes and three behaviors measured, pairs were on average more similar in the USA (mean $\mathrm{z}$-transformed $\mathrm{ICC}=.31$ ) as compared to Korea (mean $\mathrm{z}$-transformed $\mathrm{ICC}=.16), \mathrm{Z}=1.42, p=.08$. ICCs by sample are reported in the online supplements Table $\mathrm{S} 1$.

${ }^{5}$ Each of the between-nation differences remained significant when controlling for age and relationship length (see the online supplements Table S2). The one exception is the between-nation difference in length was no longer significant when controlling for age.
} 
There were also important similarities in the relationships of Americans and Koreans, as the majority of pairs in both nations defined their relationship as a friendship (the proportion of pairs sampled that identified themselves as friends did not vary by nation, USA $=54 \%$, Korea $=$ $\left.58 \%, \chi^{2}(1)=1.72, p=.19\right)$ and pairs in the USA and in Korea reported a similar degree of interpersonal closeness.

\section{Independent Self-Construal, Relational Mobility and Within-Pair Similarity}

We used multilevel structural equation modeling (MSEM) to test the direct effect of independent self-construal on within-pair similarity and the indirect effect through relational mobility, treating individuals as nested within relationship pairs (Preacher, Zyphur, \& Zhang, 2010). MSEM treats random effects as latent variables and partitions the effects into betweenpairs and within-pairs components. In this analysis, country is a pair-level indicator variable $(0=$ Korea, $1=\mathrm{USA})$, independent self-construal is an individual-level predictor variable, relational mobility is an individual-level mediator variable, and within-pair similarity as measured by the similarity index is a pair-level outcome variable. Because the outcome variable was measured only at the between level, the indirect effect only occurs at the between level (i.e., there is no within level indirect effect). Similarity index scores were reversed prior to analysis so that higher scores indicate greater similarity.

Results of this analysis are displayed in Figure 1. American pairs were higher in independent self-construal than Korean pairs $(b=1.26, S E=.09, p<.0005)$. The direct effect of independent self-construal on the similarity index was significant $(b=0.27, S E=.07, p<.0005)$. Consistent with our hypothesis, independent self-construal was associated with greater withinpair similarity. Independent self-construal was also positively associated with relational mobility, both at the between-pairs level $(b=0.90, S E=.10, p<.0005)$ and at the within-pairs level $(b=0.13$, 
$S E=.05, p=.004)$. Contrary to our hypothesis, however, relational mobility was negatively related to within-pair similarity $(b=-0.17, S E=.07, p=.02)$. The indirect effect of independent selfconstrual on similarity through relational mobility was negative and significant $(b=-0.16$, $S E=.07,95 \%$ Bayesian credible interval $=-0.22$ to -0.09$)$. Unexpectedly, after controlling for between-country differences, higher relational mobility was associated with less within-pair similarity.

Using a free-range field method we found that higher levels of independent self-construal are associated with greater similarity within relationship pairs and greater perception of relational mobility. Independent self-construal appears to support the belief that relationships are established (or dissolved) as a matter of choice, freeing people to seek out relationships that meet their personal needs and goals (Bahns et al., 2017). In a context of free choice and ample opportunity to meet new people, people prefer to form relationships with those who are like them.

By contrast, lower levels of independent self-construal are associated with less similarity within relationship pairs and lower perception of relational mobility. When relationships are viewed as products of one's environment rather than a matter of personal choice (Adams, Anderson, \& Adonu, 2004), there is less opportunity for similarity-based selection processes to influence relationship formation. Individual differences in independent self-construal translated into pair-level differences in similarity.

When comparing the data across nation, the American pairs were more similar to each other than the Korean pairs, and also higher in relational mobility. This resulted in a positive correlation between mobility and similarity. However, within each nation, the relations were reversed - for both Koreans and Americans, pairs with high beliefs in mobility had lower levels 
of similarity. This is a near-perfect demonstration of Simpson's paradox (Kievit, Frankenhuis, Waldorp \& Borsboom, 2013), where a relationship between two variables within categories or samples is reversed when subgroups are analyzed separately. This typically happens when one of the samples is higher on both variables - if the difference is sufficiently large, it can outweigh the within-sample relationship in the opposite direction (see Gardner, 1979, Wagner, 1982). The analysis considers our phenomenon simultaneously in terms of individual differences and cultural differences; we demonstrate the familiar finding that both the individual- and national/cultural-level are required to understand the processes of joining and ending relationships.

The difference between the two levels of analysis suggests further scrutiny of how relational mobility relates to similarity is needed. In Study 2, we reconceptualized relational mobility as two factors: the ease of entering relationships and the ease of exiting relationships, in a further attempt to elucidate these patterns.

\section{Study 2}

Study 2 used a self-report method to investigate the relationships between belief in friendship choice, relational mobility and friendship similarity. Based on Study 1, we hypothesized that Americans would report stronger endorsement of the importance of similarity in a friend and greater perceived similarity to friends compared to Koreans. We also hypothesized that Americans would report stronger belief in friendship choice and higher relational mobility than Koreans.

We developed a mediational model to test the direct effect of belief in friendship choice on importance and perception of friendship similarity, as well as the indirect effects through 
relational mobility ${ }^{6}$. While relational mobility is conceptualized as the opportunity to both enter and exit relationships (Schug et al., 2009; Schug, Yuki, \& Maddux, 2010), researchers have typically treated the scale as a unidimensional construct. But distinguishing entry and exit is essential—opportunity and choice may operate differently on relationship initiation and dissolution processes. An important contribution of the current research is examining the divergent effects of relational mobility on similarity-based selection processes depending on whether aspects of relationship initiation (Entry) or dissolution (Exit) are emphasized. Thomson and colleagues (2018) identified "meeting" and "choosing" as separate components of the relational mobility scale, although they do not make separate predictions about how each component influences behavior. While the "meeting/choosing" components do share some overlap with our "entry/exit" factors, there are discrepancies. Our "entry" and "exit" components highlight the aspects of relational mobility that make forming new relationships and leaving existing relationships easy or difficult.

The relative ease of entry into relationships should increase similarity in friendship choices, because ample opportunity to meet new people encourages people to be selective. When the number and variety of social choices is plentiful, it becomes easier to select friends on the basis of similarity (Bahns et al., 2012). In choosing friends who are like us, people engage in niche building to create a social environment for themselves that is safe, stable, and satisfying (Bahns et al., 2017). Yet niche building is constrained by opportunity, such that ease of initiating new relationships facilitates the process. By contrast, the relative ease of exit from relationships should decrease similarity in friendship choices because removing the fear of getting "stuck" in

\footnotetext{
${ }^{6} \mathrm{We}$ also tested willingness to dispose of social ties as an alternate mediator to relational mobility. Details are available in the online supplements.
} 
an unsatisfying relationship should relax the selection criteria. It becomes less important to find an ideal match when friendships are viewed as voluntary and exchangeable.

\section{Method}

\section{Participants}

A total of 608 participants $(N=279$ Americans and $N=329$ Koreans $)$ was recruited in Study 2. The American sample included 200 participants recruited on Amazon Mechanical Turk and 79 female undergraduates from a liberal arts college in the Northeastern region of the United States. The Korean sample included 328 participants recruited through a Facebook ad targeted at South Koreans living within 25 miles of Seoul, and one participant recruited on Amazon Mechanical Turk. The American sample included 162 women, 114 men, and 3 individuals who identified their gender as nonbinary or gender fluid. The Korean sample included 270 women, 52 men, and 7 individuals who did not report their gender. The American and Korean samples did not differ in age $(t<1 ; M=28.79$ years, $S D=8.76$, $\mathrm{MIN}=18$, MAX=67).

\section{Materials and Procedure}

All measures were assessed in English for the American sample and in Korean for the Korean sample using an online questionnaire hosted on Qualtrics or SurveyMonkey. Measures were translated into Korean using the back translation method.

Belief in friendship choice. Four items ( $\alpha$ s=.71, Korean; .76, American) assessed beliefs about how much choice people have in determining who their friends are. The items were "People can choose who their friends are," "If I think about who my friends are, it would be fair to say that I chose to be friends with them," "If I meet someone whom I do not like, I can usually decide not to interact with that person again," and "I do not have control over who my friends are [reverse scored]" (1=Strongly disagree, 7=Strongly agree $)$. Higher scores reflect stronger 
endorsement of the belief in friendship choice. While belief in friendship choice is correlated with relational mobility, a principal components analysis confirmed they are separate factors (with no cross-loadings greater than .25).

Importance of similarity. Eight items ( $\alpha \mathrm{s}=.81$, Korean; .83, American) assessed participants' beliefs about how important it is that their friends are similar in attitudes, values, preferences, habits, goals, and humor. Example items include "It is important to me that my friends hold similar attitudes to my own" and "It is important to me that my friends have the same likes and dislikes as I do" (1=Strongly disagree, 7=Strongly agree). Higher scores reflect stronger endorsement of the importance of similarity.

Perceived similarity. Eight items $(\alpha \mathrm{s}=.82$, Korean; .84, American) assessed how similar participants perceived themselves and their closest friends to be in attitudes, values, preferences, habits, goals, and humor. Example items include "Overall, my friends and I hold similar values" and "Overall, my friends and I have similar goals in life" (1=Strongly disagree, 7=Strongly agree). Higher scores reflect greater perceived similarity.

Relational mobility. Relational mobility was assessed with Thomson and colleagues’ (2018) relational mobility scale. Twelve items ( $\alpha s=.82$, Korean; .87 American) assessed perceptions of how easily people in the immediate society ("your school, workplace, town, neighborhood, etc.”) can freely enter and exit social relationships. Based on the results of a principal components analysis with Varimax rotation, the scale was divided into separate factors for relative ease of relationship entry ( 8 items, $\alpha s=.81$, Korean; .84 , American) and exit (4 items, $\alpha s=.67$, Korean; .83, American). All items had primary factor loadings greater than .4 and crossloadings less than .4 (see online supplements Table S3). The Entry factor explained $31 \%$ of the variance, and the Exit factor explained $23 \%$ of the variance in responses to the relational 
mobility scale. The Entry factor reflects the shared belief that it is easy to meet new people who may be potential friends. The Exit factor reflects the shared belief that if people were not satisfied with their current relationships, they would be free to leave. Higher scores reflect greater ease of relationship entry and exit, respectively.

\section{Results and Discussion}

\section{Tests of Between-Country Differences}

A series of independent samples t-tests compared American and Korean participants on all constructs measured; results are presented in Table 3. Americans reported stronger endorsement of the importance of similarity in a friend and greater perceptions of similarity to their friends than Koreans, which is consistent with our hypothesis and with the findings of Study 1. Also as hypothesized, Americans reported stronger endorsement of the belief in friendship choice and greater relational mobility than Koreans including greater ease of relationship entry and exit.

\section{Belief in Friendship Choice, Relational Mobility, and Friendship Similarity}

Two multiple mediator models were estimated using PROCESS Model 4 (Hayes, 2013) in SPSS to test the direct effect of belief in friendship choice on the rated importance of similarity $^{7}$ in a friend as well as the indirect effects through the two components of the relational mobility scale: Entry and Exit. The model shown in Figure 2a combines the American and Korean samples and does not control for cultural context. As a point of comparison, the model shown in Figure $2 \mathrm{~b}$ does control for cultural context by including country $(0=$ Korea, $1=\mathrm{USA})$ as a covariate. Confidence intervals for tests of indirect effects were generated using 5000 bootstrap samples.

\footnotetext{
${ }^{7}$ The findings were replicated using perceived similarity as the outcome measure. Details are available in the online supplements.
} 
As shown in Figure 2a, belief in friendship choice was positively correlated with ease of relationship entry $(b=0.41 S E=.04, p<.0005)$. Easy entry was, in turn, correlated with increased importance of similarity $(b=0.28, S E=.05, p<.001)$. The indirect effect of belief in friendship choice on importance of similarity through the Entry factor was positive and significant $(b=0.11$, $S E=.02,95 \% \mathrm{CI}=.07$ to .17$)$.

Belief in friendship choice was also positively correlated with ease of relationship exit $(b=0.39, S E=.04, p<.0005)$. Easy exit was, in turn, correlated with reduced importance of similarity $(b=-0.22, S E=.05, p<.0005)$. The indirect effect of belief in friendship choice on importance of similarity through the Exit factor was negative and significant $(b=-0.08, S E=.02$, $95 \% \mathrm{CI}=-.14$ to -.04$)$. The total effect was positive and significant $(b=0.24, S E=.04, p<.0005)$. After controlling for the indirect effects through relational mobility (Entry and Exit), the direct effect remains significant but its magnitude is somewhat reduced $(b=0.21, S E=.05, p<.0005)$.

As shown in Figure 2b, all of the relationships remain the same when controlling for cultural context but the magnitude of the regression weights is somewhat reduced. This model reveals that the effects of relational mobility on similarity are in part due to between-country differences. Americans reported greater relational mobility in the Entry $(b=.70, S E=.08, p<.0005)$ but not the Exit component $(b=.12, S E=.08, p=.13)$, and greater importance of similarity $(b=.79$, $S E=.09, p<.005)$ compared to Koreans. Importantly, even after controlling for these betweencountry differences, belief in friendship choice remains a significant predictor of friendship similarity both directly and indirectly through relational mobility.

These findings make clear that relational mobility is a two-dimensional construct, with the Entry and Exit components each bearing a different relationship to friendship similarity. Endorsement of the relative ease of relationship entry reflects the belief that there are many 
opportunities to meet new people and that people can choose with whom they interact. A sense of ample opportunity and choice in relationships invites people to be selective in deciding with whom they spend their time. On the other hand, easy exit from relationships reflects the belief that one can freely choose to leave unsatisfying relationships. Just as the belief that one could get "stuck" in an unsatisfying relationship may increase the urgency of selecting a well-matched friend, so too does the belief that unsatisfying relationships are easy to get out of relax the importance of similarity-based selection criteria.

\section{Study 3}

In Study 3 we manipulated the relative ease of relationship entry and exit using only American participants. We sought to replicate the pattern of effects identified in Study 2, manipulating beliefs about relationship entry and exit in the USA as a model for the betweencountry differences observed in Studies 1 and 2 (Betancourt et al., 1992). Based on the rationale presented in Study 2 , we hypothesized easy entry would increase the importance of similarity in deciding whether to pursue a friendship, because ample opportunity to meet new people should invite people to be selective in choosing friends. By contrast, we hypothesized that easy exit from relationships would decrease the importance of similarity, because removing the fear of "getting stuck" in an undesirable relationship should relax the selection criteria.

\section{Method}

\section{Participants and Design}

American participants $(N=199)$ were recruited on Amazon Mechanical Turk. About 10\% of the sample ( $n=19)$ was excluded for having completed the study in less than 30 seconds, which we deemed was too quick to have carefully read the materials. This left a sample of 180 participants for analysis. The majority of the sample $(61 \%, n=110)$ was male, $37 \%$ were female 
$(n=66), 1 \%(n=2)$ did not identify as either male or female, and 1\% $(n=2)$ did not report gender. Participants ranged in age from 19 to 65 years $(M=33.14, S D=9.62)$. The experiment used a 2 (ease of relationship entry: high, low) x 2 (ease of relationship exit: high, low) between-subjects design.

\section{Materials and Procedure}

Participants first read the experimental manipulation which asked them to consider how they would respond as someone interested in making new friends. The manipulation varied the anticipated ease of Entry into and Exit from friendship with the following text: "In this life, you've found that you often/rarely have the opportunity to make friends with someone who is interested in you as a friend. Because of the nature of your life and social circle, it's fairly easy/difficult to end a friendship if you no longer find it satisfying."

Next participants completed the dependent measure. ${ }^{8}$ Four items $(\alpha=.72)$ measured Importance of Similarity, with participants indicating what would be important in deciding whether to pursue a new friendship. The items were "It would be important to me that they hold similar attitudes/values to my own," and "It would be important to me that they enjoy the same things I do/have the same likes and dislikes as me." Higher scores reflect greater importance of similarity. All items were assessed on a 1 (Strongly disagree) to 5 (Strongly agree) scale. All study materials were hosted on Qualtrics.

\section{Results and Discussion}

A 2 (ease of entry: high, low) x 2 (ease of exit: high, low) between-subjects ANOVA was conducted on the importance of similarity (see Table 4). As predicted, there was a significant effect of exit such that ease of exit reduced the importance of similarity in a friend,

\footnotetext{
${ }^{8}$ We also measured general interest in making new friends which was unrelated to Entry or Exit. Details are available in the online supplements.
} 
$F(1,176)=4.24, p=.04, d=.29$. Participants reported that they cared less about whether a potential friend was similar to them when the anticipated ease of exit from a friendship was high $(M=3.91$, $S D=0.69)$ compared to low $(M=4.09, S D=0.52)$. Contrary to our hypothesis, there was no effect of ease of entry on importance of similarity, $F(1,176)=0.32, p=.57, d=.10$. The interaction of entry and exit also was not significant, $F(1,176)=1.25, p=.27 .{ }^{9}$

These findings highlight how the desire for similarity in a friend is affected by expectations about the relative ease or difficulty of ending a friendship. When one anticipates that ending a friendship will be easy, there is less need to be highly selective in choosing friends. Similarity is a reliable predictor of attraction and liking, but such assurances of compatibility are not critical when unsatisfying friendships can easily be dissolved. By contrast, when one anticipates the possibility of "getting stuck" in an unsatisfying friendship, one is more inclined to choose friends carefully. Similarity-related information may be more valuable in a context where ending a friendship can be precarious.

We did not find any effects for the entry manipulation in this American context where individuals perceive a high degree of choice in determining who their friends are. It is possible that our American participants' shared cultural beliefs about relationship choice made the suggestion that opportunities to make new friends are rare seem implausible. Further research is needed to test the causal relationship between ease of entry and increased importance of similarity.

\section{General Discussion}

\footnotetext{
${ }^{9}$ We conducted a nearly identical experiment in the context of romantic relationships and found no significant effects of the manipulations of entry and exit on importance of similarity (see online supplements). This suggests that the relationships we have identified between relational mobility and similarity may be specific to the context of friendship.
} 
Americans reported greater opportunity than Koreans to select and maintain relationships as a matter of personal choice, and American relationship partners were more similar and endorsed the importance of similarity in friendship more than Koreans. But perceived choice and similarity were negatively related within nations_-a Simpson's paradoxical finding which led us to suppose that ease of entry and ease of exit might have opposite effects on similarity.

We propose that beliefs about choice and a related sense of relational mobility determine how we go about choosing friends. Similarity is important in both cultures (Heine et al., 2009; Schug et al., 2009), but similarity plays a key role mostly when it is possible to choose. Similarity-based selection processes are stronger, so friends and partners are more similar, in contexts that offer a greater sense of relationship choice.

Our methods detected differences between two distinct cultural contexts, but they also detected differences within cultures by measuring individual beliefs about relationships. Independent self-construal encourages the belief that close relationships are formed to satisfy personal preferences. Study 1 showed how independent self-construal facilitates similarity-based selection processes in relationship formation. People who were high in independent selfconstrual reported a greater perception of relational mobility and were more similar to the relationship partners we found them with than people who were low in independent selfconstrual. Surprisingly, however, in Study 1 greater relational mobility was negatively related to similarity. These results suggest that the relationship between relational mobility and similarity is more complex than previously realized.

Relational mobility matters, but it matters differently for relationship entry and exit in our samples. Relational mobility research has typically conflated formation and termination (Schug et al., 2009; Schug et al., 2010), and our Study 1 confounded the two components as well. 
Importantly, in Study 2 we broke down the relational mobility scale into separate components for relationship entry and exit, and these dimensions revealed different effects on the rated importance of similarity in friendship. Ease of entry, the ample opportunity to meet new people and ability to decide about whom to interact with, invites people to be selective. By contrast easy exit, with full expectation of being able to leave an unsatisfying relationship relaxes the pressure to be selective. Our findings in regard to ease of relationship exit are consistent with work by Chen, Chiu, and Chan (2009) demonstrating that the perceived importance of fit or compatibility of a prospective employee is increased in contexts of low job mobility (see also Bahns et al., 2012).

In Study 3, we replicated the effect of easy exit reducing the importance of similarity criteria in an experiment. In an environment of easy exit, similarity-based selection criteria seem unnecessary as there is no fear of "getting stuck" with an incompatible friend. We did not replicate the effect of easy entry increasing the importance of similarity, perhaps because the manipulation of relationship entry was less effective in an American sample. Variability of choice and opportunity among contexts is key to understanding whether relationship entry and exit are independent contributors to the importance of similarity. Our data provide only a first look at the question.

Some researchers have found (Morry, 2005, 2007; Murray, Holmes, Bellavia, Griffin, \& Dolderman, 2002) that actual or perceived similarity is associated with greater relationship satisfaction in Western samples (cf. Finkel, Eastwick, Karney, Reis, \& Sprecher, 2012). However, we are not suggesting that the lower degree of similarity means the Korean friendships are any less satisfying. Closeness between American and Korean samples did not differ in Study 1, and Bahns et al. (2012) found that American friends at small colleges characterized by low 
relational mobility were less similar, but more close, than friends at a large state university characterized by high mobility and greater choice. Despite being less similar, friendships in lowchoice environments may be equally satisfying. Friendships in low-choice settings are more stable due to barriers to relationship entry and exit, and stability itself may promote closeness and satisfaction (Rusbult, 1983).

We used three different methods to explore the relationships between beliefs about choice, relational mobility and similarity of relationship choices, each with its own set of strengths and limitations. The clear advantage of the field method (Study 1) is in objectively measuring similarity within pairs, by comparing the responses of both members of the pair. Another strength is that we recruited participants from several different cities in each country, making the findings more representative of each cultural context than they would have been if all the data were collected from a single location and increasing the observed variance in key constructs. The diversity of our samples was dependent on the locations where the data were collected, and so the pairs we sampled were on average older and the relationships were more longstanding in the American samples. It is unlikely that this finding reflects systematic differences in the friendships found in each country, but is almost certainly tied to the locations from which we sampled.

A strength and limitation of the field method is that it captures relationships of all kinds, rather than exclusively sampling friendships. A majority of relationships we sampled were friendships, and the findings in Study 1 were not moderated by relationship type, but we think that our data is best understood in the context of friendship. In Studies 2 and 3 the dependent measure of the importance of similarity was tailored to the context of friendship. 
The field method in Study 1 dictated that the questionnaire be kept short, and as a result we constructed quite short versions of scales. We chose an indicator variable strategy, which maximizes "breadth" of measurement but reduces intercorrelations among individual items; the strategy maximizes validity but suppresses apparent reliability (Bollen, 1984). It also meant we were unable to adequately measure the separate components of relationship entry and exit in Study 1 . This constrains our confidence over the negative relationship identified between relational mobility and pair similarity from Study 1.

\section{Constraints on Generality}

The "cultural context" of this research crosses geographical lines, but it is not geographical lines that determine cultural comparisons; it is a comparison across internal representations of choice, relationships, values, and construals that we have studied. The effects are not so simple as a cross-national comparison; our effects must be researched by also studying different people in the same geography, whose representations and construals differ in measurable ways.

Our findings provide evidence of the relationships between independent self-construal, relational mobility, and the importance of similarity in friendship identified in American and Korean younger adults. We expect the results to generalize to younger adults' friendships in other nations as well, provided that similar measures of independent self-construal and relational mobility are used, sufficient variability on these constructs is observed, and that choosing friends is not fully constrained by culture or other external constraints. The domain of similarity measured should include attitudes, values, habits, or preferences that are important for friendship in the relevant cultural context. For Study 1's field method, it is important that pairs do not discuss their answers while filling out the questionnaires. For Study 3, the manipulation of 
relative ease/difficulty of entry and exit must be believable in the relevant cultural context. Unpublished data from our lab found the effect of the exit manipulation was not replicated in the context of romantic relationships; this effect may not generalize to non-friendship contexts. We have no reason to believe that the results depend on other characteristics of the participants, materials, or context.

\section{Conclusion}

Selecting friends on the basis of similarity is facilitated by contexts that promote independent self-construal and a sense of relationship choice. Perceiving ample choice and opportunity to form new friendships may increase the utility of similarity-based selection, while anticipating easy exit from existing relationships relaxes the importance of similarity. Individual differences in beliefs about relationship choice constrain the magnitude of the similarityattraction effect. Feeling that we have a choice about friends makes us choosy, and we can afford to like mostly the people who are like us. 


\section{References}

Adams, G., Anderson, S. L., \& Adonu, J. K. (2004). The cultural grounding of closeness and intimacy. In D. J. Mashek \& A. P. Aron (Eds.), Handbook of closeness and intimacy. (pp. 321-339). Mahwah, NJ: Lawrence Erlbaum Associates Publishers.

Bahns, A. J., Crandall, C. S., Gillath, O., \& Preacher, K. J. (2017). Similarity in relationships as niche construction: Choice, stability, and influence within dyads in a free choice environment. Journal of Personality and Social Psychology, 112(2), 329-355. doi: $10.1037 / \mathrm{pspp} 0000088$

Bahns, A. J., Pickett, K. M., \& Crandall, C. S. (2012). Social ecology of similarity: Big schools, small schools and social relationships. Group Processes \& Intergroup Relations, 15(1), 119-131. doi:10.1177/1368430211410751

Bellah, R., Madsen, R., Sullivan, W., Swidler, A., \& Tipton, S. (1985). Habits of the heart: Individuialism and commitment in American life. Berkeley, CA: University of California Press.

Betancourt, H., Hardin, C., \& Manzi, J. (1992). Beliefs, value orientation, and culture in attribution processes and helping behavior. Journal of Cross-Cultural Psychology, 23(2), 179-195. doi:10.1177/0022022192232004

Bollen, K. A. (1984). Multiple indicators: Internal consistency or no necessary relationship?. Quality and Quantity, 18(4), 377-385.

Bourgeois, M. J., \& Bowen, A. (2001). Self-organization of alcohol-related attitudes and beliefs in a campus housing complex: An initial investigation. Health Psychology, 20, 434-437. doi:10.1037/0278-6133.20.6.434

Byrne, D. (1971). The attraction paradigm. New York: Academic Press. 
Chen, J., Chiu, C.-y., \& Chan, S. F. (2009). The cultural effects of job mobility and the belief in a fixed world: Evidence from performance forecast. Journal of Personality and Social Psychology, 97(5), 851-865. doi:10.1037/a0015950

Crandall, C. S., Schiffhauer, K. L., \& Harvey, R. (1997). Friendship pair similarity as a measure of group value. Group Dynamics: Theory, Research, and Practice, 1(2), 133-143. doi:10.1037/1089-2699.1.2.133

Cross, S. E., Bacon, P. L., \& Morris, M. L. (2000). The relational-interdependent self-construal and relationships. Journal of Personality and Social Psychology, 78, 791-808. doi:10.1037/0022-3514.78.4.791

Cross, S. E., \& Gore, J. S. (2004). The relational self-construal and closeness. In D. J. Mashek \& A. P. Aron (Eds.), Handbook of closeness and intimacy. (pp. 229-245). Mahwah, NJ, US: Lawrence Erlbaum Associates Publishers.

Cullum, J., \& Harton, H. C. (2007). Cultural evolution: Interpersonal influence, issue importance, and the development of shared attitudes in college residence halls. Personality and Social Psychology Bulletin, 33, 1327-1339. doi:10.1177/0146167207303949

Derlega, V. J., \& Chaikin, A. L. (1977). Privacy and self-disclosure in social relationships. Journal of Social Issues, 33(3), 102-115. doi:10.1111/j.1540-4560.1977.tb01885.x

Finkel, E. J., Eastwick, P. W., Karney, B. R., Reis, H. T., \& Sprecher, S. (2012). Online dating: A critical analysis from the perspective of psychological science. Psychological Science in the Public Interest, 13, 3-66. doi:10.1177/1529100612436522

Gardner, M. (1979). Mathematical games: On the fabric of inductive logic, and some probability paradoxes. Scientific American, 234 (March), 119-122, 124. 
Gillath, O., \& Keefer, L. A. (2016). Generalizing disposability: Residential mobility and the willingness to dissolve social ties. Personal Relationships, 23(2), 186-198. doi:10.1111/pere.12119

Graziano, W. G., \& Bruce, J. W. (2008). Attraction and the initiation of relationships: A review of the empirical literature. In S. Sprecher, A. Wenzel, \& J. H. Harvey (Eds.), Handbook of relationship initiation (pp. 269-298). New York: Psychology Press.

Heine, S. J., Foster, J.-A. B., \& Spina, R. (2009). Do birds of a feather universally flock together? Cultural variation in the similarity-attraction effect. Asian Journal of Social Psychology, 12(4), 247-258. doi:10.1111/j.1467-839X.2009.01289.x

Heine, S. J., \& Renshaw, K. (2002). Interjudge agreement, self-enhancement, and liking: Crosscultural divergences. Personality and Social Psychology Bulletin, 28(5), 578-587. doi:10.1177/0146167202288002

Hofstede, G. (1991). Cultures and organizations: Software of the mind. London, England: McGraw-Hill.

Ishiguro, I. (2011). Attitude homophily and relational selectability: An analysis of dyadic data. The Japanese Society of Social Psychology. Japanese Journal of Social Psychology, 27(1), 13-23. doi/10.14966/jssp.KJ00007406646

Jones, J. T., Pelham, B. W., Carvallo, M., \& Mirenberg, M. C. (2004). How do I love thee? Let me count the Js: Implicit egotism and interpersonal attraction. Journal of Personality and Social Psychology, 87, 665-683.

Kagitcibasi, C. (2005). Autonomy and relatedness in cultural context: Implications for self and family. Journal of Cross-Cultural Psychology, 36(4), 403-422. doi:10.1177/0022022105275959 
Kashima, Y., Yamaguchi, S., Kim, U., Choi, S.-C., Gelfand, M. J., \& Yuki, M. (1995). Culture, gender, and self: A perspective from individualism-collectivism research. Journal of Personality and Social Psychology, 69(5), 925-937. doi:10.1037/0022-3514.69.5.925

Kievit, R., Frankenhuis, W. Waldorp, L. \& Borsboom, D. (2013). Simpson's paradox in psychological science: A practical guide. Frontiers in Psychology, 4(513), 1-14.

Kim, U. (1994). Individualism and collectivism: Conceptual clarifiaction and elaboration. In U. Kim, H. C. Triandis, C. Kagitcibasi, S.-C. Choi, \& G. Yoon (Eds.), Individualism and collectivism: Theory, method, and applications (pp. 19-40). Thousand Oaks, CA: Sage.

Kim, K. I., Park, H. J., \& Suzuki, N. (1990). Reward allocations in the United States, Japan, and Korea: A comparison of individualistic and collectivistic cultures. Academy of Management Journal, 33, 188-198.

Markus, H. R., \& Kitayama, S. (1991). Culture and the self: Implications for cognition, emotion, and motivation. Psychological Review, 98(2), 224-253. doi:10.1037/0033-295X.98.2.224

McPherson, M., Smith-Lovin, L., \& Cook, J. M. (2001). Birds of a feather: Homophily in social networks. Annual Review of Sociology, 27, 415-444. doi:10.1146/annurev.soc.27.1.415

Morry, M. M. (2005). Relationship satisfaction as a predictor of similarity ratings: A test of the attraction-similarity hypothesis. Journal of Social and Personal Relationships, 22(4), 561-584. doi:10.1177/0265407505054524

Morry, M. M. (2007). The attraction-similarity hypothesis among cross-sex friends: Relationship satisfaction, perceived similarities, and self-serving perceptions. Journal of Social and Personal Relationships, 24(1), 117-138. doi:10.1177/0265407507072615 
Murray, S. L., Holmes, J. G., Bellavia, G., Griffin, D. W., \& Dolderman, D. (2002). Kindred spirits? The benefits of egocentrism in close relationships. Journal of Personality and Social Psychology, 82(4), 563-581. doi:10.1037/0022-3514.82.4.563

Muthén, B., \& Asparouhov, T. (2012). Bayesian structural equation modeling: A more flexible representation of substantive theory. Psychologiacl Methods, 17(3), 313-335. doi:10.1037/a0026802

Newcomb, T. M. (1956). The prediction of interpersonal attraction. American Psychologist, 11(11), 575-586. doi:10.1037/h0046141

Oyserman, D. (1993). The lens of personhood: Viewing the self and others in a multicultural society. Journal of Personality and Social Psychology, 65(5), 993-1009. doi:10.1037/0022-3514.65.5.993

Oyserman, D., Coon, H. M., \& Kemmelmeier, M. (2002). Rethinking individualism and collectivism: Evaluation of theoretical assumptions and meta-analyses. Psychological Bulletin, 128(1), 3-72. doi:10.1037//0033-2909.128.1.3

Preacher, K. J., Zyphur, M. J., \& Zhang, Z. (2010). A general multilevel SEM framework for assessing multilevel mediation. Psychological Methods, 15, 209-233.

Rusbult, C. E. (1983). A longitudinal test of the investment model: The development (and deterioration) of satisfaction and commitment in heterosexual involvements. Journal of Personality and Social Psychology, 45(1), 101-117. doi:10.1037/0022-3514.45.1.101

Schug, J., Yuki, M., Horikawa, H., \& Takemura, K. (2009). Similarity attraction and actually selecting similar others: How cross-societal differences in relational mobility affect interpersonal similarity in Japan and the USA. Asian Journal of Social Psychology, 12(2), 95-103. doi:10.1111/j.1467-839X.2009.01277.x 
Schug, J., Yuki, M., \& Maddux, W. (2010). Relational mobility explains between- and withinculture differences in self-disclosure to close friends. Psychological Science, 21(10), 1471-1478. doi:10.1177/0956797610382786

Thibaut, J. W., \& Kelley, H. H. (1959). The social psychology of groups. New York: John Wiley. Thomson, R., Yuki, M., Talhelm, T., Schug, J., Kito, M., Ayanian, A. H., ... \& Ferreira, C. M. (2018). Relational mobility predicts social behaviors in 39 countries and is tied to historical farming and threat. Proceedings of the National Academy of Sciences, 115(29), 7521-7526. doi: 10.1073/pnas.1713191115

Titzmann, P. F., Silbereisen, R. K., \& Mesch, G. S. (2012). Change in friendship homophily: A German Israeli comparison of adolescent immigrants. Journal of Cross-Cultural Psychology, 43(3), 410-428. doi:10.1177/0022022111399648

Triandis. (1995). Individualism and collectivism. Boulder, CO: Westview Press.

Urberg, K. A., Degirmencioglu, S. M., \& Tolson, J. M. (1998). Adolescent friendship selection and termination: The role of similarity. Journal of Social and Personal Relationships, 15(5), 703-710. doi:10.1177/0265407598155008

Wagner, C. H. (1982). Simpson's paradox in real life. The American Statistician, 36, 46-48.

Watson, D., Klohnen, E. C., Casillas, A., Simms, E. N., \& Haig, J. (2004). Match makers and deal breakers: Analyses of assortative mating in newlywed couples. Journal of Personality and Social Psychology, 72, 1029-1068. doi:10.1111/j.00223506.2004.00289.x

Wiseman, J. (1986). Friendship: Bonds and binds in a voluntary relationship. Journal of Social and Personal Relationships, 3, 191-211. 
Yuki, M., \& Schug, J. (2012). Relational mobility: A socioecological approach to personal relationships. In O. Gillath, G. Adams, \& A. Kunkel (Eds.), Relationship Science: Integrating Evolutionary, Neuroscience, and Sociocultural Approaches. (pp. 137-151). Washington, DC, US: American Psychological Association.

Zhang, R., \& Li, L. M. W. (2014). The acculturation of relational mobility: An investigation of Asian Canadians. Journal of Cross-Cultural Psychology, 45(9), 1390-1410.

doi: $10.1177 / 0022022114542850$ 


\section{Table 1}

Descriptive Statistics by Sample, Study 1

\begin{tabular}{|c|c|c|c|c|c|c|}
\hline & \multicolumn{4}{|c|}{ USA } & \multicolumn{2}{|c|}{ Korea } \\
\hline & New York & Chicago & Iowa City & Lawrence & Seoul & Gongju \\
\hline Population size & $8,405,837$ & $2,695,598$ & 67,862 & 87,643 & $10,143,645$ & 116,369 \\
\hline Population density (people $/ \mathrm{km}^{2}$ ) & $6,921.52$ & $4,391.44$ & $1,036.54$ & 987.75 & $16,739.85$ & 134.76 \\
\hline Sample size ( $N$ individuals) & 192 & 98 & 56 & 160 & 150 & 140 \\
\hline \multicolumn{7}{|l|}{ Cultural Variables } \\
\hline Relational Mobility $\dagger$ & $5.00^{\ddagger}(1.10)$ & $5.69(1.06)$ & $5.72(0.87)$ & $5.50(1.02)$ & $4.60^{\ddagger}(1.00)$ & $3.80(1.09)$ \\
\hline Independent self-construal $\dagger$ & $5.50(1.24)$ & $5.57(1.03)$ & $5.53(1.09)$ & $5.55(1.06)$ & $4.25(1.12)$ & $4.34(1.16)$ \\
\hline \multicolumn{7}{|l|}{ Relationship Descriptors } \\
\hline Degree of Liking $\dagger$ & $6.60(0.87)$ & $6.74(0.61)$ & $6.67(0.67)$ & $6.60(0.803)$ & $6.17(1.24)$ & $6.14(1.02)$ \\
\hline Closeness & $6.16(1.39)$ & $6.15(1.34)$ & $6.07(1.44)$ & $6.01(1.45)$ & $6.07(1.53)$ & $6.13(1.13)$ \\
\hline Degree of Disclosure $\dagger$ & $5.91(1.47)$ & $6.28(1.16)$ & $5.91(1.39)$ & $5.89(1.53)$ & $5.79(1.39)$ & $5.61(1.39)$ \\
\hline Length of Relationship $\dagger$ & $72.90^{*}(100.19)$ & $112.85(148.66)$ & $69.88(99.71)$ & $111.71(122.31)$ & $59.26(69.75)$ & $46.08(52.37)$ \\
\hline Hours Spent Together $\dagger$ & $46.33^{\ddagger}(52.42)$ & $30.51(34.91)$ & $39.13(41.96)$ & $25.81(35.01)$ & $13.31^{\ddagger}(18.20)$ & $18.70(25.87)$ \\
\hline \multicolumn{7}{|l|}{ Attitudes } \\
\hline Political Beliefs $\dagger$ & $5.17^{\ddagger}(1.65)$ & $4.68(1.83)$ & $5.36(1.41)$ & $4.60(1.72)$ & $4.16^{\ddagger}(1.27)$ & $4.44(1.18)$ \\
\hline Protestant Work Ethic & $5.59(1.41)$ & $5.85(1.45)$ & $5.66(1.52)$ & $5.70(1.33)$ & $5.55(1.26)$ & $5.83(1.29)$ \\
\hline
\end{tabular}




\begin{tabular}{|c|c|c|c|c|c|c|}
\hline & \multicolumn{4}{|c|}{ USA } & \multicolumn{2}{|c|}{ Korea } \\
\hline & New York & Chicago & Iowa City & Lawrence & Seoul & Gongju \\
\hline United States Relations $\dagger$ & $3.81(1.78)$ & $3.77(1.84)$ & $3.42(1.87)$ & $3.74(1.63)$ & $4.36(1.11)$ & $4.19(1.46)$ \\
\hline North Korea Relations $\dagger$ & $4.18^{\dagger}(1.52)$ & $3.60(1.47)$ & $3.84(1.23)$ & $3.91(1.18)$ & $3.54(1.36)$ & $3.44(1.60)$ \\
\hline College Attitudes $\dagger$ & $4.85 \div(1.60)$ & $4.87(1.84)$ & $4.16(1.75)$ & $4.55(1.68)$ & $4.47 \div(1.58)$ & $3.95(1.74)$ \\
\hline Religion Attitudes & $5.21 \div(1.94)$ & $5.22(1.97)$ & $5.62(1.87)$ & $4.64(2.02)$ & $5.02 \div(1.90)$ & $5.55(1.69)$ \\
\hline Abortion Attitudes $\uparrow$ & $5.63^{\dagger}(1.79)$ & $4.92(2.43)$ & $5.77(2.00)$ & $4.76(2.17)$ & $4.12(1.77)$ & $3.81(1.39)$ \\
\hline South Korean Prejudice $\dagger$ & $5.09^{\ddagger}(1.29)$ & $5.34(1.44)$ & $5.71(1.40)$ & $5.31(1.32)$ & $5.41(1.17)$ & $5.59(1.18)$ \\
\hline North Korean Prejudice† & $4.59^{\dagger}(1.33)$ & $4.65(1.58)$ & $5.16(1.62)$ & $4.64(1.49)$ & $3.59(1.36)$ & $3.81(1.39)$ \\
\hline American Prejudice† & $5.11^{\ddagger}(1.24)$ & $5.34(1.38)$ & $5.88(1.16)$ & $5.62(1.20)$ & $4.63(1.10)$ & $4.69(1.16)$ \\
\hline Fat Prejudice $\dagger$ & $4.73(1.33)$ & $4.73(1.62)$ & $5.20(1.59)$ & $4.82(1.61)$ & $3.87(1.17)$ & $4.08(1.38)$ \\
\hline Gay Prejudice $\dagger$ & $5.07^{\ddagger}(1.51)$ & $5.61(1.59)$ & $6.02(1.23)$ & $5.10(1.60)$ & $3.40(1.52)$ & $3.07(1.75)$ \\
\hline \multicolumn{7}{|l|}{ Health Behaviors } \\
\hline Smoking Tobacco $\dagger$ & $2.24^{\dagger}(1.98)$ & 2.07 (1.92) & $2.96(2.34)$ & $1.94(1.75)$ & $1.53^{\ddagger}(1.40)$ & $2.00(2.02)$ \\
\hline Drinking Alcohol $\dagger$ & $3.18^{\dagger}(1.94)$ & $2.93(1.94)$ & 4.39 (1.96) & $2.71(1.77)$ & $2.32(1.45)$ & $2.51(1.72)$ \\
\hline Exercising $\dagger$ & $4.00^{\dagger}(1.80)$ & $4.36(1.74)$ & $4.57(1.70)$ & $3.83(1.78)$ & $3.05(1.85)$ & $3.34(1.86)$ \\
\hline
\end{tabular}

Note. Tabled values are means; standard deviations are in parentheses.

$\uparrow$ Between-country comparison, samples significantly differ at $p<.05$.

${ }^{\star}$ Within-country comparison, samples significantly differ at $p<.05$. 


\section{Table 2}

Comparing Relationship Pairs in the USA and Korea, Study 1

\begin{tabular}{lllll}
\hline & \multicolumn{1}{c}{ American } & \multicolumn{1}{c}{ Korean } & & \\
\cline { 2 - 3 } & \multicolumn{1}{c}{$M(S D)$} & $M(S D)$ & \multirow{2}{*}{$t(d f)$} & $d$ \\
\cline { 2 - 4 } Similarity index & $0.52(0.18)$ & $0.66(0.23)$ & $6.50(252)^{* * *}$ & 0.68 \\
Relational mobility & $5.37(1.08)$ & $4.21(1.12)$ & $14.34(793)^{* * *}$ & 1.05 \\
Independent self-construal & $5.53(1.13)$ & $4.29(1.13)$ & $14.88(793)^{* * *}$ & 1.10 \\
Age & $30.79(13.53)$ & $23.83(5.97)$ & $9.99(753)^{* * *}$ & 0.67 \\
Length of relationship & $93.86(119.70)$ & $52.85(62.16)$ & $6.29(769)^{* * *}$ & 0.43 \\
Degree of liking & $6.64(0.78)$ & $6.16(1.14)$ & $6.33(449)^{* * * *}$ & 0.49 \\
Hours spent together & $36.10(44.06)$ & $15.80(22.20)$ & $8.43(749)^{* * *}$ & 0.58 \\
Degree of disclosure & $5.98(1.43)$ & $5.70(1.39)$ & $2.67(793)^{* * *}$ & 0.20 \\
Closeness & $6.10(1.40)$ & $6.10(1.35)$ & $0.04(776)$ & 0.00 \\
\hline
\end{tabular}

Note. Similarity index reflects the mean discrepancy score across 12 attitudes and 3 health behaviors for each dyad. The t-test statistic for similarity index is calculated from $N=398$ dyads and uses adjusted degrees of freedom because equal variances are not assumed. $* p<.05$. $* * p<.01 . * * * p<.001$. 


\section{Table 3}

Descriptive Statistics and Correlation Matrix for American and Korean Samples, Study 2

\begin{tabular}{|c|c|c|c|c|c|c|c|c|c|}
\hline & American & Korean & & & & & & & \\
\hline & $M(S D)$ & $M(S D)$ & $t(d f)$ & $d$ & 1. & 2. & 3. & 4. & 5. \\
\hline 1. RM_Entry & $5.10(0.94)$ & $4.22(0.90)$ & $11.75(605)^{* * *}$ & 0.96 & -- & $.50 * * *$ & -.04 & .03 & $.17 * *$ \\
\hline 2. RM_Exit & $4.56(1.15)$ & $4.21(0.90)$ & $4.17(604)^{* * *}$ & 0.34 & $.55 * * *$ & -- & -.11 & -.06 & $.18^{* *}$ \\
\hline 3. Importance of similarity & $4.79(0.89)$ & $3.88(0.99)$ & $11.94(604) * * *$ & 0.97 & $.17 * *$ & -.06 & -- & $.67 * * *$ & .08 \\
\hline 4. Perceived similarity & $5.19(0.84)$ & $4.02(0.90)$ & $16.43(604)^{* * *}$ & 1.33 & $.27 * * *$ & .01 & $.66^{* * *}$ & -- & .04 \\
\hline 5. Belief in friendship choice & $5.93(0.92)$ & $5.30(0.93)$ & $8.34(603)^{* * *}$ & 0.68 & $.46^{* * *}$ & $.49 * * *$ & .12 & $.25 * * *$ & -- \\
\hline
\end{tabular}

Note. Values in numbered columns are correlations; values above the diagonal are for Korea and below the diagonal for USA. RM_Entry=relational mobility Entry factor. RM_Exit=relational mobility Exit factor. ${ }^{*} p<.05 .{ }^{* *} p<.01 .{ }^{* * *} p<.001$. 


\section{Table 4}

Importance of Similarity by Ease of Entry and Exit, Study 3

\begin{tabular}{|c|c|c|c|c|c|c|c|c|c|c|c|}
\hline & & \multicolumn{10}{|c|}{ Ease of Exit } \\
\hline & & \multicolumn{3}{|c|}{ Low } & \multicolumn{4}{|c|}{ High } & \multicolumn{3}{|c|}{ Total } \\
\hline & & $N$ & $M$ & $S D$ & $N$ & $M$ & $S D$ & $d$ & $N$ & $M$ & $S D$ \\
\hline \multirow[t]{3}{*}{ Ease of Entry } & Low & 45 & 4.02 & 0.51 & 47 & 3.93 & 0.72 & 0.14 & 92 & 3.97 & 0.63 \\
\hline & High & 44 & 4.17 & 0.52 & 44 & 3.88 & 0.66 & 0.49 & 88 & 4.03 & 0.61 \\
\hline & Total & 89 & 4.09 & 0.52 & 91 & 3.91 & 0.69 & 0.29 & 180 & 4.00 & 0.62 \\
\hline
\end{tabular}


Figure 1

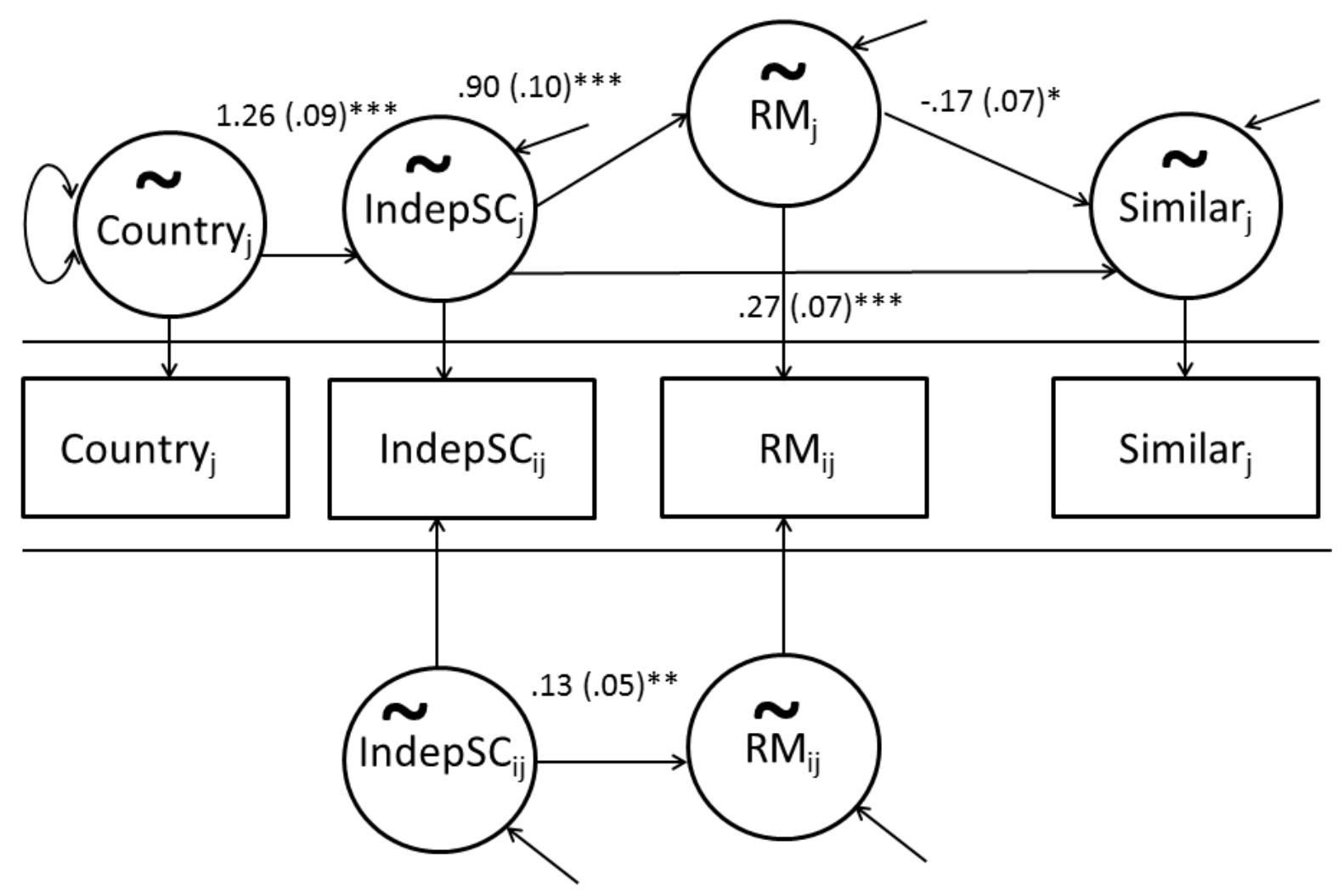

Figure 1. A multilevel structural equation model tested the indirect effect of independent selfconstrual on pair similarity through relational mobility. Subscript $i$ indicates construct varies between individuals and subscript $j$ indicates construct varies between dyads. Country is a categorical indicator variable coded $0=$ Korea, $1=$ USA. IndepSC $=$ independent self-construal. $\mathrm{RM}=$ relational mobility. Similar=similarity index. Paths labeled with unstandardized regression coefficients; standard errors in parentheses. ${ }^{*} p<.05, * * p<.01, * * * p<.001$. 
Figures $2 \mathrm{a}$ and $2 b$
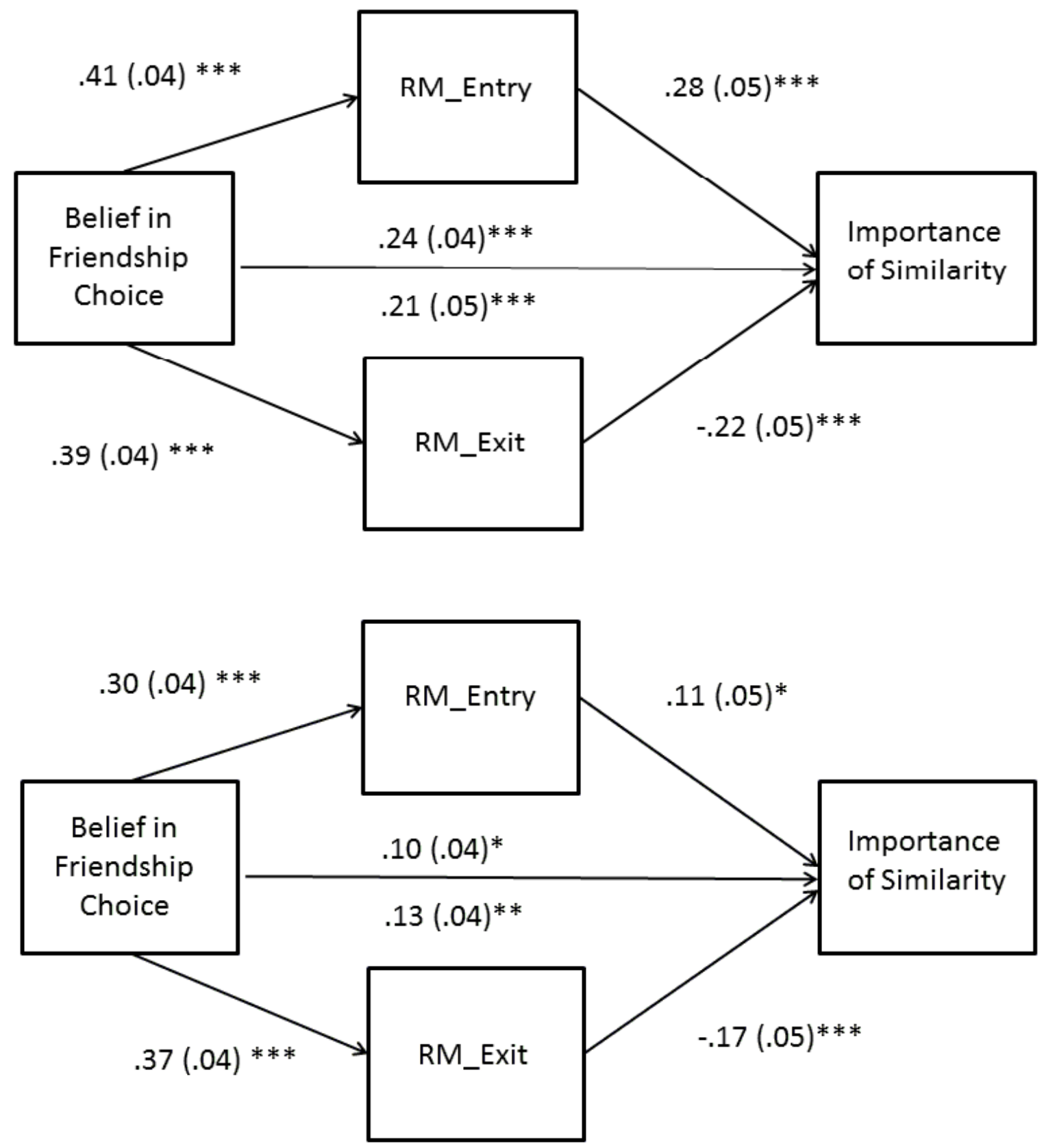

Figures $2 a$ and $2 b$. Multiple mediator models tested the indirect effect of belief in friendship choice on importance of similarity through ease of relationship entry and exit. Fig. 2a (top panel) does not control for cultural context. Figure $2 b$ (bottom panel) includes Country $(0=$ Korea, 1=USA) as a covariate. RM_Entry=relational mobility Entry factor. RM_Exit=relational mobility Exit factor. Paths labeled with unstandardized regression coefficients; standard errors in parentheses. ${ }^{*} p<.05, * * * p<.005$. 